\title{
Modes on the Standard and Inverted Conductor-Backed Slotline
}

\author{
Jan Zehentner, Jan Machac, Jan Mrkvica, Carlo Tuzi \\ Czech Technical University in Prague, Technicka 2, 16627 Prague 6, Czech Republic
}

\begin{abstract}
This paper recalls modes which can propagate on the conductor-backed slotline. The characteristic of the dominant mode on the CBSL is documented. The ICBSL is a modification of the CBSL placed in a dielectric with permittivity higher than the substrate permittivity. The modes on this line, including the interesting dominant leaky mode, are presented.
\end{abstract}

\section{INTRODUCTION}

The standard slotline (SL) has been known for many years and has frequently been applied in circuits such as the hybrid branch-coupler, hybrid ring coupler, filter, and mode transformer. Its behaviour in a wide frequency band has been studied in detail, and a more or less complete analysis is available.

The dominant bound mode with even symmetry of the transversal component of the electric field within the slot is utilized for signal transmission. The first odd space leaky mode enables the design of an antenna radiating into space on both sides of the antenna. The requirement to radiate power into the half-space drew our attention to the conductor-backed slotline (CBSL) with the cross-section shown in Fig. 1, where the first medium is air. It turned out that, besides the known even and odd modes leaking power in the substrate, even and odd non-leaking dominant modes can also occur on this line [1]. In this paper we would like to provide evidence of the existence of the even dominant mode both by our own observations and by the measurement presented in [2].

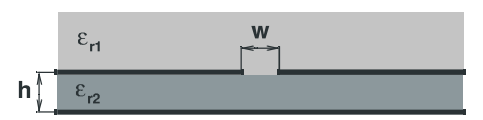

Fig. 1 Cross-section of the conductor-backed slotline when $\varepsilon_{\mathrm{r} 1}<\varepsilon_{\mathrm{r} 2}$ and the inverted conductor-backed slotline when $\varepsilon_{\mathrm{r} 1}>\varepsilon_{\mathrm{r} 2}$.

In addition, we introduce new findings concerning modes on the CBSL when the substrate permittivity is less than the permittivity of the surrounding space. A crosssection of this line is shown in Fig. 1, where $\varepsilon_{\mathrm{r} 1}>\varepsilon_{\mathrm{r} 2}$. Since the ratio of the permittivities of the outer and inner dielectric is now inverted in comparison to the CBSL case, we will refer to this line as the inverted conductor-backed slotline (ICBSL).
In $[1,3,4]$, we presented solutions of the dispersion equation representing even and odd modes belonging to the CBSL. Since the simplest slot excitation is by a loop across the slot terminating the coaxial line, we will further consider even modes. A feature of the field in particular parts of the SL, CBSL, ICBSL cross-sections can be explained by the total reflection concept.

The SL consists of two conductors, and the CBSL and ICBSL consist of three conductors. Their dominant modes must approach the quasi TEM mode when the frequency decreases to zero. The dominant mode on the SL is the bound mode. The effective permittivity of the small substrate area in the close vicinity of the slot is higher than outside this area. Consequently, the field amplitude in the substrate decreases laterally.

\section{CONDUCTOR-BACKED SLOTLINE}

On the other hand, the effective permittivity in the central part of the CBSL cross-section is less than the substrate permittivity at a greater distance from the slot, and is higher than the permittivity of the air above the line. Consequently the dominant mode in air decreases with distance from the slot, and in the substrate a standing wave has to occur laterally.

The dispersion characteristics of the even dominant mode and the first mode leaking in the substrate of the CBSL calculated by the method of moments in the spectral domain were published in $[1,3,4]$. The calculated electric field distribution of the even dominant mode on the cross-sectional plane is plotted in Fig. 2. The monotonic and periodic character of the field in air and in the substrate is evident.

The backward Fourier transform determines an instantaneous value of the electric field in the space domain, i. e. also its component in the substrate perpendicular to the metal plates $\mathrm{E}_{\mathrm{y} 2}(\mathrm{x}, \mathrm{y})$. Only integration along the real axis $\xi$ of the real part of the integrand $\operatorname{Re}\left[\mathrm{F}(\xi, \mathrm{y})_{\mathrm{x}}\right]=$ $\operatorname{Re}\left[\tilde{\mathrm{E}}_{\mathrm{y} 2}(\xi, \mathrm{y}) \cdot \mathrm{e}^{-\mathrm{j} \xi \mathrm{x}}\right]$ is accounted for, as its imaginary part is the odd function of $\xi$. $\operatorname{Re}\left[\mathrm{F}(\xi, \mathrm{y})_{\mathrm{x}}\right]$, plotted in Fig. 3, is the odd function of $\xi$ with respect to the poles position $\xi_{0}$ and is therefore integrated in the sense of V. P. (valeur principale). The result of integration represented by $\mathrm{E}_{\mathrm{y} 2}(\mathrm{x}, \mathrm{y})$ is drawn in Fig. 2 with a typical oscillating char- 


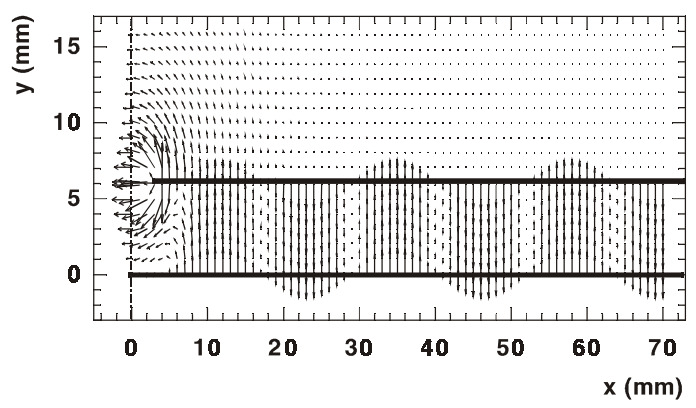

Fig. 2 Distribution of the electric field of the even dominant mode on the transversal plane of the CBSL when $\mathrm{w}=6 \mathrm{~mm}, \mathrm{~h}=6$ $\mathrm{mm}, \varepsilon_{\mathrm{r} 2}=2.6, \varepsilon_{\mathrm{r} 1}=1$ and $\mathrm{h} / \lambda_{0}=0.3$.

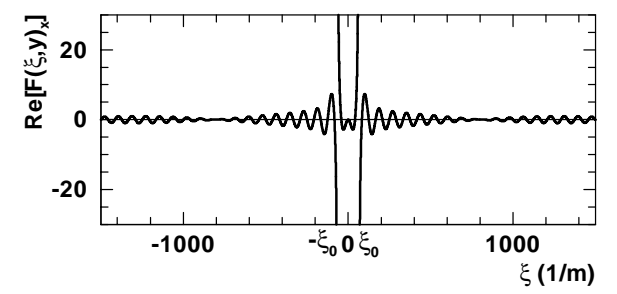

Fig. 3 Dependence of the real part of the backward Fourier transform integrand $\operatorname{Re}\left[\mathrm{F}(\xi, \mathrm{y})_{\mathrm{x}}\right]$ on $\xi$ when $\mathrm{x}=72.475 \mathrm{~mm}$, $\mathrm{y}=\mathrm{h} / 2=3 \mathrm{~mm}, \varepsilon_{\mathrm{r} 2}=2.6, \varepsilon_{\mathrm{r} 1}=1$.

acter. The correctness of the integration has been proved by another integration scheme proposed by Mosig in [5]. The first term of $\left\{\operatorname{Re}\left[\mathrm{F}(\xi, \mathrm{y})_{\mathrm{x}}\right]-\operatorname{Res}\left[\operatorname{Re}\left[\mathrm{F}\left(\xi_{0}, \mathrm{y}\right)_{\mathrm{x}}\right]\right] /\left(\xi_{-} \xi_{0}\right)\right\}+$ $\operatorname{Res}\left[\operatorname{Re}\left[\mathrm{F}\left(\xi_{0}, \mathrm{y}\right)_{\mathrm{x}}\right]\right] /\left(\xi_{-} \xi_{0}\right)$ extracts the singularity and the second term is integrated analytically, Res denotes residue. The result is identical with that in Fig. 2. The integration can therefore be considered correct from the mathematical and numerical point of view.

The wave equation solved in the sourceless region provides the field of possible modes which may exist on the guiding line. That is our case. The even dominant mode could therefore excite on the CBSL alone from dc up to the frequency $f_{c}$ at which the 1 st mode leaking in the substrate sets up, as is shown in Fig. 7. At the cut-off $f_{c}$, the propagation constant $\beta / \mathrm{k}_{0}=\sqrt{ } \varepsilon_{\mathrm{r} 2}$. It is well known that the real source excites a germ of the leaky mode inside the frequency gap. This then gradually evolves with growing frequency into a full-blooded leaky mode, typical above the pertinent cut-off [6]. Interval $\left(0, f_{c}\right)$ is a special frequency gap $\Delta \mathrm{f}$ in our case.

We simulated the field under various excitations. A delta-gap source across the slot provides at zero frequency on a boundless CBSL between parallel plates a constant electric field out of phase in the left and right region. Inside our $\Delta \mathrm{f}$, in the substrate, a delta-gap excites the electric field $\mathrm{E}_{\mathrm{y} 2}$ shown in Fig. 4. Energy propagates in all directions with decreasing amplitude, but in the four directions determined by characteristic angles it has the highest magnitude, even from the lowest frequency. Conversely, a theoretically regular leaky wave sets up from the zero leakage angle. Computed propagation constant $\beta$ in Fig. 8 is real corresponding to an ideally unbounded line on which the dominant mode may exist. It is lower than the propagation constant of the TEM parallel plate mode. Consequently, the phase condition for a rise in leakage is met. However, we can speak only about a „,conditionally leaky mode”.

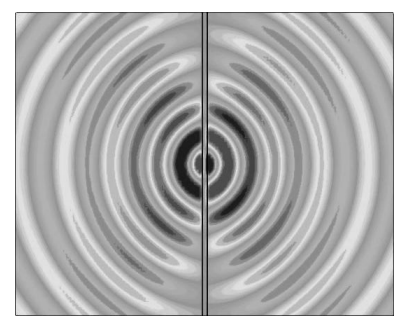

Fig. 4 Electric field component in the CBSL substrate perpendicular to the metal plates at $3 \mathrm{GHz}$ excited by the deltagap source.

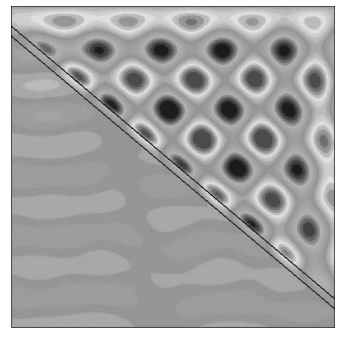

a

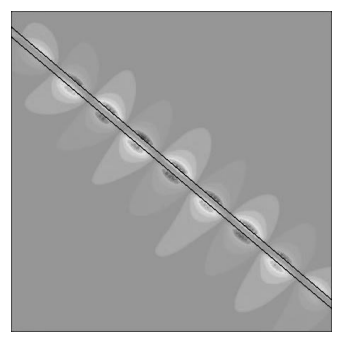

b
Fig. 5 Electric field component in the CBSL substrate perpendicular to metal plates at $5 \mathrm{GHz}$ excited by a uniform source placed at the edge of the substrate and in air (b) above the CBSL also perpendicular to the metal plates.

Fig. 5a shows the rise in the electric field in the substrate (a) when a TEM wave is incident at the angle $\vartheta=\arccos \left(\beta / k_{0} \sqrt{ } \varepsilon_{\mathrm{r} 2}\right)$ to the region below the slot. The source is uniform along the top edge of the substrate. The total field is a wave propagating along the slot. Laterally it has the character of a standing wave. The field does not pass to the area on the opposite side of the slot but is coupled though the slot to air, where the wave propagating along the slot appears as shown in Fig. 5b. The same effect occurs when discrete delta-gap sources are placed between parallel plates, perpendicular to the line axis, spaced periodically with an alternated adjoining phase shift. 
The electric field measured at the edge of the front cross-section of the line substrate $93 \mathrm{~cm}$ away from the excitation point at $2.25 \mathrm{GHz}$ shown in Fig. 6 confirms the presence of the dominant mode and the absence of the leaky mode. The calculated and measured wavelength of the standing wave in the lateral direction is $107.2 \mathrm{~mm}$ and $105.4 \mathrm{~mm}$, respectively. The measured propagation constant of the dominant and the 1st leaky modes are compared with the calculated dispersion characteristics in Fig. 7.

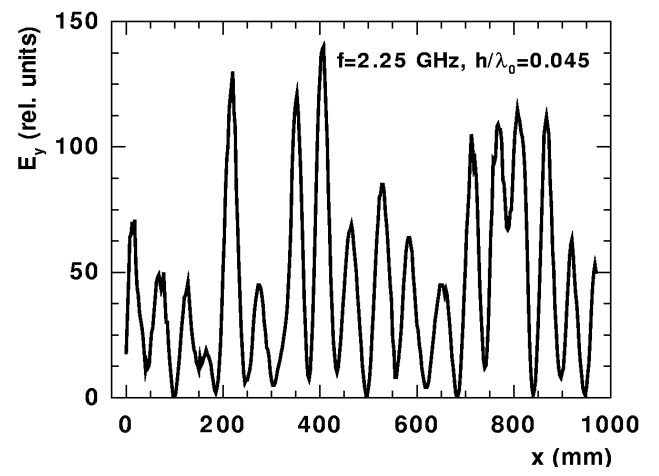

Fig. 6 Electric field component perpendicular to the conductors of the CBSL from Fig. 3 measured along the front edge of the substrate dielectric slab at $2.25 \mathrm{GHz}$ when $\mathrm{h} / \lambda_{0}=0.45$.

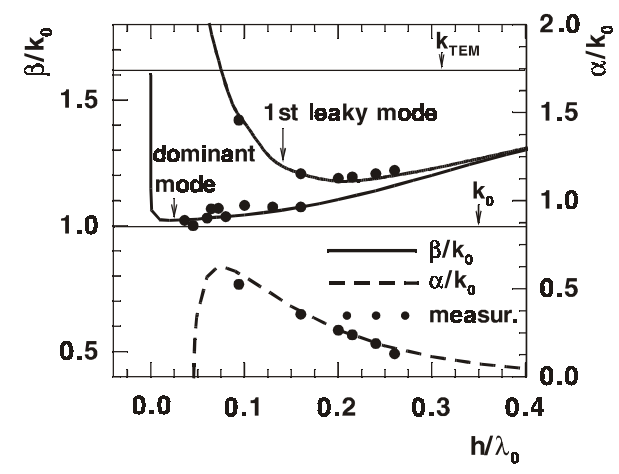

Fig. 7 Measured and calculated dispersion characteristics of the dominant and the 1st even leaky modes on the CBSL from Fig. 2.

Why did we observe the dominant mode on the CBSL with finite size of the substrate? From the excitation point the field spreads as in Fig. 4. It reflects from the back and side walls of the substrate and is incident under various angles to the region below the slot. These reflections supply absence of the source at infinity. The resultant field propagates along the slot in air and in the substrate. It forms a ,standing" wave laterally in the substrate. Note that the wavelength of this ,standing” wave varies slightly due to the different angles of incidence, as is also confirmed by the measurement.

At frequencies higher than $\mathrm{f}_{\mathrm{c}}$ the even dominant mode and the 1st mode leaking in the substrate propagate simultaneously $[1,3,4]$. This fact was evident already in the record presented in [2], one half of which is reproduced here in Fig. 8. The calculated and measured wavelengths of the standing wave in the transversal direction are $31.9 \mathrm{~mm}$ and $30.7 \mathrm{~mm}$, respectively. Our measurement presented in [4] confirmed the same phenomenon.

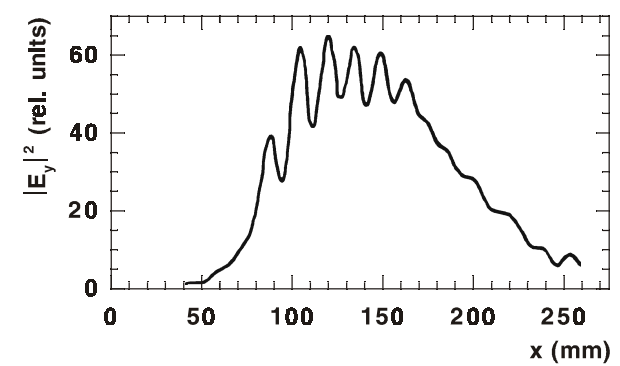

Fig. 8 Reproduction of one half of the measured leakage pattern in Fig 5, published in [2].

\section{INVERTED CONDUCTOR-BACKED SLOTLINE}

The ICBSL is the line with modes that are potentially able to leak power into the space above the line, since their phase constants are lower than $\mathrm{k}_{1}=\sqrt{ } \varepsilon_{\mathrm{r} 1} \mathrm{k}_{0}$. Moreover, since $\varepsilon_{\mathrm{r} 1}>\varepsilon_{\mathrm{r} 2}$ the ICBSL is able to support a mode leaking in the space which does not simultaneously leak in the substrate where this mode is bound. This behaviour occurred in the newly revealed dominant leaky mode with even symmetry of the transversal electric field component within the slot. The dispersion characteristic of this mode and the other even mode leaking simultaneously in the space and in the substrate are plotted in Fig. 9. The attenuation constant of the dominant leaky mode decreases to zero with the fall in frequency.

The electric field distribution of the dominant mode, evolves with the frequency increase from the quasi TEM mode with the field uniformly distributed to infinity within the substrate to the field bound to the slot in the substrate and leaking energy in the space shown in Fig. 10. According to the generalized condition of leakage [7] no mode leaking in the substrate is physical on the ICBSL. Its

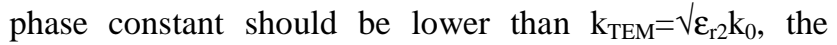
phase constant of the TEM mode propagating in the parallel plate waveguide, and at the same time higher than $\mathrm{k}_{1}=\sqrt{ } \varepsilon_{\mathrm{r} 1} \mathrm{k}_{0}$. However, since $\varepsilon_{\mathrm{r} 1}>\varepsilon_{\mathrm{r} 2}$, this condition cannot be met. 


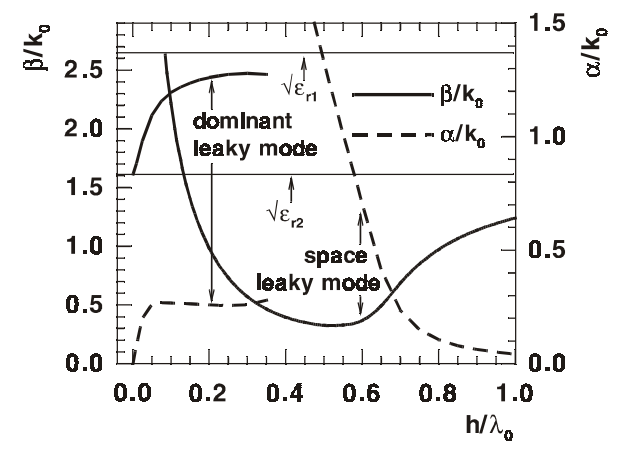

Fig. 9 Dispersion characteristics of even modes on the ICBSL with $\mathrm{w}=6 \mathrm{~mm}, \mathrm{~h}=6 \mathrm{~mm}, \varepsilon_{\mathrm{r} 1}=7$ and $\varepsilon_{\mathrm{r} 2}=2.6$.

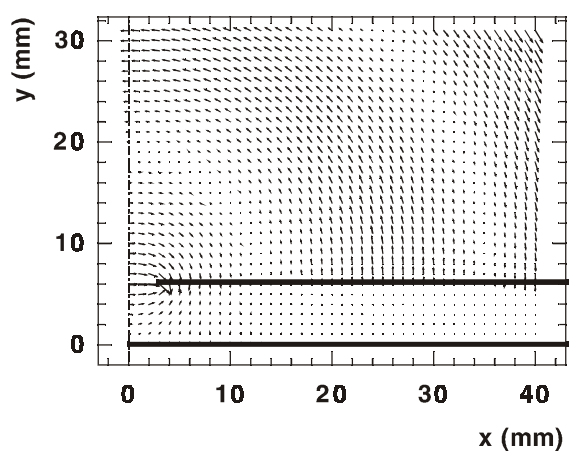

Fig. 10 Computed electric field distribution of the even dominant leaky mode on the transversal plane of the ICBSL from Fig. 9 at $\mathrm{h} / \lambda_{0}=0.1(5 \mathrm{GHz})$.

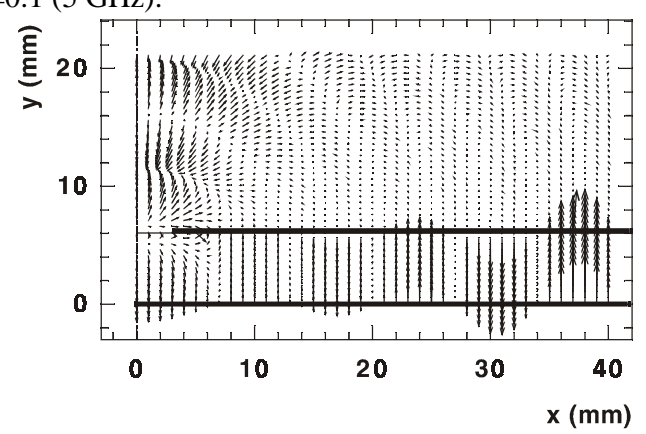

Fig. 11 Computed electric field of the odd leaky mode on the transversal plane of the ICBSL with $\mathrm{w}=10 \mathrm{~mm}, \mathrm{~h}=6 \mathrm{~mm}, \varepsilon_{\mathrm{r} 1}=7$ and $\varepsilon_{\mathrm{r} 2}=2.6$ at $\mathrm{h} / \lambda_{0}=0.3(15 \mathrm{GHz})$.

In addition, we identified standard odd space leaky modes on the ICBSL. Their dispersion characteristics will be presented at the symposium. Unlike the field of the dominant leaky mode shown in Fig. 10, the field of these modes also grows laterally in the substrate, as is seen in Fig.11.

\section{CONCLUSION}

This paper gives evidence about the existence of the dominant mode on the conductor-backed slotline. This mode propagates along the line unattenuatedly from zero frequency and possesses a standing wave character in the lateral direction in the substrate. The field distribution of this mode was confirmed by measuring it along the front substrate edge. This paper introduces the inverted conductor-backed slotline and some modes propagating along this line. This is promising for leaky wave antenna applications. We have revealed on this line a dominant leaky mode which can be excited from zero frequency as a quasi TEM wave, and which propagates with no attenuation and transforms gradually into a space leaky wave with increasing frequency. The dispersion characteristics, and the field distribution of the odd space leaky modes on the ICBSL, will be introduced during the presentation.

\section{ACKNOWLEDGMENTS}

This work has been supported by the Grant Agency of the Czech Republic under project 102/03/0449 and by research program J04/98 212300014.

\section{REFERENCES}

[1] J. Zehentner, J. Machac, J. Mrkvica, "Novel selected modes on the conductor-backed slotline" 2002 IEEE MTT-S Int. Microwave Symp. Dig., vol. 2, pp. 961964, June 2002.

[2] H. Shigesawa, M. Tsuji, A. A. Oliner, "Conductorbacked slot line and coplanar waveguide: danger and full-wave analyses," 1988 IEEE MTT-S Int. Microwave Symp. Dig., vol. 1, pp. 199-202, June 1988.

[3] J. Zehentner, J. Machac, J. Mrkvica, "Even and odd modes on a conductor-backed slotline, " Proc. of the 32nd European Microwave Conference, vol. 2, pp. 609-612, September 2002.

[4] J. Zehentner, J. Machac, J. Mrkvica, J. Hruska, V. Langer, J. Zabloudil, "Experimental verification of theoretically revealed modes on the conductor-backed slotline," 2002 APMC Proc., vol. 2, pp. 1200-1203, November 2002.

[5] T. Itoh Ed., Numerical Techniques for Microwave and Millimeter-Wave Passive Structures, New York: John Wiley \& Sons, 1989.

[6] W. L. Langston, J. T. Williams, D. R. Jackson, F. Mesa, "Frequency dependent characteristics of radiation from a voltage source on a covered microstrip line" 2002 IEEE MTT-S Int. Microwave Symp. Dig., vol. 2, pp. 949-952, June 2002.

[7] C. Di Nallo, F. Mesa, D. R. Jackson, "Excitation of leaky modes on multilayer stripline structures," IEEE Trans. Microwave Theory and Tech., vol. MTT-46, no. 8, pp. 1062-1071, August 1998. 